$\begin{gathered}\text { Revista do Departamento de Geografia } \\ \text { Universidade de São Paulo } \\ \text { www.revistas.usp.br/rdg }\end{gathered}$
V.31 (2016)
ISSN 2236-2878

\title{
A CLASSIFICAÇÃO FLUVIAL DE ROSGEN APLICADA EM CÓRREGOS DA REGIÃO OESTE DO ESTADO DO PARANÁ, BRASIL
}

\author{
THE APPLICATION OF THE ROSGEN METHOD TO STREAMS OF THE \\ WESTERN OF THE STATE OF PARANÁ, BRAZIL
}

\author{
Oscar Vicente Quinonez Fernandez \\ Universidade Estadual do Oeste do Paraná \\ oscar.fernandez@unioeste.br
}

Recebido (Received): 10/06/2015 Aceito (Accepted): 10/02/2016
DOI: http://dx.doi.org/10.11606/rdg.v31i0.98837

Resumo: O artigo apresenta a aplicação da classificação de Rosgen em córregos da região Oeste do estado do Paraná, inserida no planalto basáltico da Bacia do Paraná. A classificação tem quatro níveis de detalhamento: caracterização morfológica básica (nível I), descrição morfológica (nível II), levantamento das condições de estabilidade do rio (nível III) e verificação (nível IV). No nível I, a classificação divide os canais em nove tipos principais (Aa+, A, B, C, E, F, G, D e DA). No nível II, a classificação prevê a divisão dos nove tipos citados em 94 subtipos baseado no padrão fluvial, grau de entrincheiramento do canal, relação largura/profundidade, índice de sinuosidade, declividade do fluxo e material de fundo. Neste trabalho foi aplicado o nível II da classificação por representar um instrumento útil para inventariar as condições morfológicas e sedimentológicas dos cursos fluviais de uma região. Dentre os 18 trechos estudados, 12 foram agrupados nos subtipos E4, E5 e G5c. Nos seis canais restantes não foi possível aplicar a classificação de Rosgen, pela falta de enquadramento das variáveis sinuosidade e declividade dentro dos limites da classificação. Estas variáveis mostraram valores abaixo dos limites da classificação em 50\% dos trechos levantados com respeito a sinuosidade e $11 \%$ na declividade. A classificação mostrou suas limitações nos córregos do planalto basáltico paranaense na aplicação da variável sinuosidade, cujos baixos índices podem ser favorecidos por lineamentos tectônicos presentes nas rochas basáltica.

Palavras-chave: Classificação fluvial; Geomorfologia fluvial; Margens plenas; Córregos de planalto basáltico.
Abstract: The article presents data collected from 18 stream sections to determine the applicability of the Rosgen Stream Classification System to the basalt plateau of the Parana basin in the Western region of the State of Paraná, Brazil. This classification has four levels of detail: broad morphological characterization (level I), morphological description (level II), stream conditions of stability (level III) and verification (level IV). The classification at level I divides the channels into 9 main types (Aa+, A, B, C, E, F, G, D and DA). Classification at level II provides 94 subtypes, based on the following parameters: channel pattern, entrenchment ratio, width/depth ratio, sinuosity, slope flow and channel material types. In this work we applied the classification at level II because it represents a useful tool to inventory the morphological and sedimentological conditions of the waterways of a region. Among the 18 studied stretches, 12 were classified as subtypes E4, E5 and G5c. In the remaining six channels it was not possible to apply the Rosgen classification due to the lack of consistency of some morphological variables within the classification limits. Among the variables used for the classification, sinuosity and slope flow showed values below the classification limits in $50 \%$ and $11 \%$ of the analyzed stretches, respectively. The classification has shown its limitations in streams of basalt plateau in the application of sinuosity, which low rates are favored by tectonic lineaments in volcanic rocks.

Keywords: Stream classification; Stream geomorphology; Bankfull; Basaltic plateau streams. 


\section{INTRODUÇÃO}

O rio constitui o objeto de estudo de engenheiros, hidrólogos, geomorfólogos, biólogos, geólogos e ecólogos. Um dos primeiros passos destes profissionais é tentar classificar os cursos fluviais de acordo com suas necessidades. Na geomorfologia fluvial, um dos objetivos da classificação de canais fluviais é reduzir as complexas unidades de estudos em unidades discretas que facilitem a compreensão.

A série de propostas de classificação de cursos fluviais começou com o trabalho de Davis (1899) e continuou com os trabalhos de Horton (1945) e Strahler (1964). No Brasil, a metodologia de hierarquização de bacias proposta por Pfafstetter (1989) tem uma ampla aceitação, sendo aplicada também em outros países sul-americanos (RUIZ et al., 2006; CRESPO et al., 2008; ANTÚNEZ, 2011 entre outros).

Goodwin (1999) organizou as principais propostas de classificação de canais fluviais divulgadas desde a metade do século XX em dois grupos, baseados na abordagem adotada pelos autores. No primeiro grupo, os autores baseiam as classificações na descrição morfológica dos rios. A mais conhecida deste grupo é a classificação de Leopold e Wolman (1957) que discrimina os canais em tipos retos, meândricos e entrelaçados. Outras propostas como as de Schumm (1963, 1981), Popov (1964), Kellerhals et al. (1976), Rust (1978), Brice (1984), Church (1992), Rosgen (1994) e Kondolf et al. (2003) integram o primeiro grupo. As classificações englobadas no segundo grupo levam em contam, além dos aspectos morfológicos do canal, os processos erosivos e deposicionais atuantes no canal e na planície de inundação. Neste grupo podem ser citados os trabalhos de Simon (1989), Whiting e Bradley (1993), Woolfe e Balzary (1996), Miall (1996) e Montgomery e Buffington (1997).

Dentre os sistemas de classificação de canais fluviais divulgados nas últimas décadas, destaca-se a proposta do hidrólogo americano David L. Rosgen. O sistema de classificação é baseado em observações realizadas em centenas de rios de vários tamanhos distribuídos em diferentes regiões climáticas da América do Norte e Nova Zelândia. As observações de campo de Rosgen começaram em 1973 e após uma década de estudos, apresentou um texto preliminar da classificação à comunidade científica (ROSGEN, 1985). Os ajustes necessários na classificação foram apresentados na versão definitiva publicada alguns anos depois (ROSGEN, 1994).

Desde a publicação da versão final, a classificação vem ganhando adeptos nos EUA e Canadá. A adoção da classificação por parte de agências governamentais de ambos os países em projetos de estabilização ou restauração de cursos d'água tornou-se frequente a partir dos anos noventa. Atualmente, a classificação de Rosgen é, sem dúvida, a mais utilizada (WARD et al., 2008). Dentre os autores que adotaram a classificação de Rosgen podem ser citados Castro (1997), Savery et al. (2001), Esptein (2002), McCandless (2003), Jones e Candler (2005), Hey (2006), Roper et al. (2008) entre outros. No Brasil, a classificação de Rosgen foi adotada por Estiliano (2006) e Almeida Neto (2007).

\section{OBJETIVO E ÁREA DE ESTUDO}

Os objetivos deste trabalho é comentar os princípios da classificação de Rosgen e discutir os resultados da aplicação desta proposta em córregos localizados nas bacias do rio Piquiri e Paraná III, região Oeste do Estado do Paraná (Figura 1). Optou-se pela classificação de Rosgen porque constitui uma proposta de ampla aceitação na comunidade científica internacional na caracterização morfológica e sedimentológica dos cursos fluviais.

A região Oeste do Paraná está inserida no terceiro planalto paranaense, onde aflora rochas basálticas da Formação Serra Geral que constitui uma província magmática que recobre $12 \times 10^{5} \mathrm{~km}^{2}$ da bacia sedimentar do Paraná e cobre porções da região centro-sul do Brasil e parte dos territórios do Paraguai, Uruguai e Argentina (NARDY et al., 2002). O terceiro planalto paranaense é dividido em sub-unidades morfoesculturais definidas por planaltos, cujo relevo regional é caracterizado por um grau de dissecação média e alta, topos alongados com cristas e, vertentes convexas e retilíneas (SANTOS et al., 2006). A origem dos planaltos da bacia do Paraná está relacionada com a evolução da Plataforma Sulamericana. O levantamento epirogenético da Plataforma ocorrida desde o Cretáceo Superior até o limite Paleógeno-Neógeno (FRANCO-MAGALHÃES et al., 2010) soergueu a região Oeste do Paraná até altitudes de $700 \mathrm{~m}$, submetendo a área de estudo a um continuo processo de dissecação. 


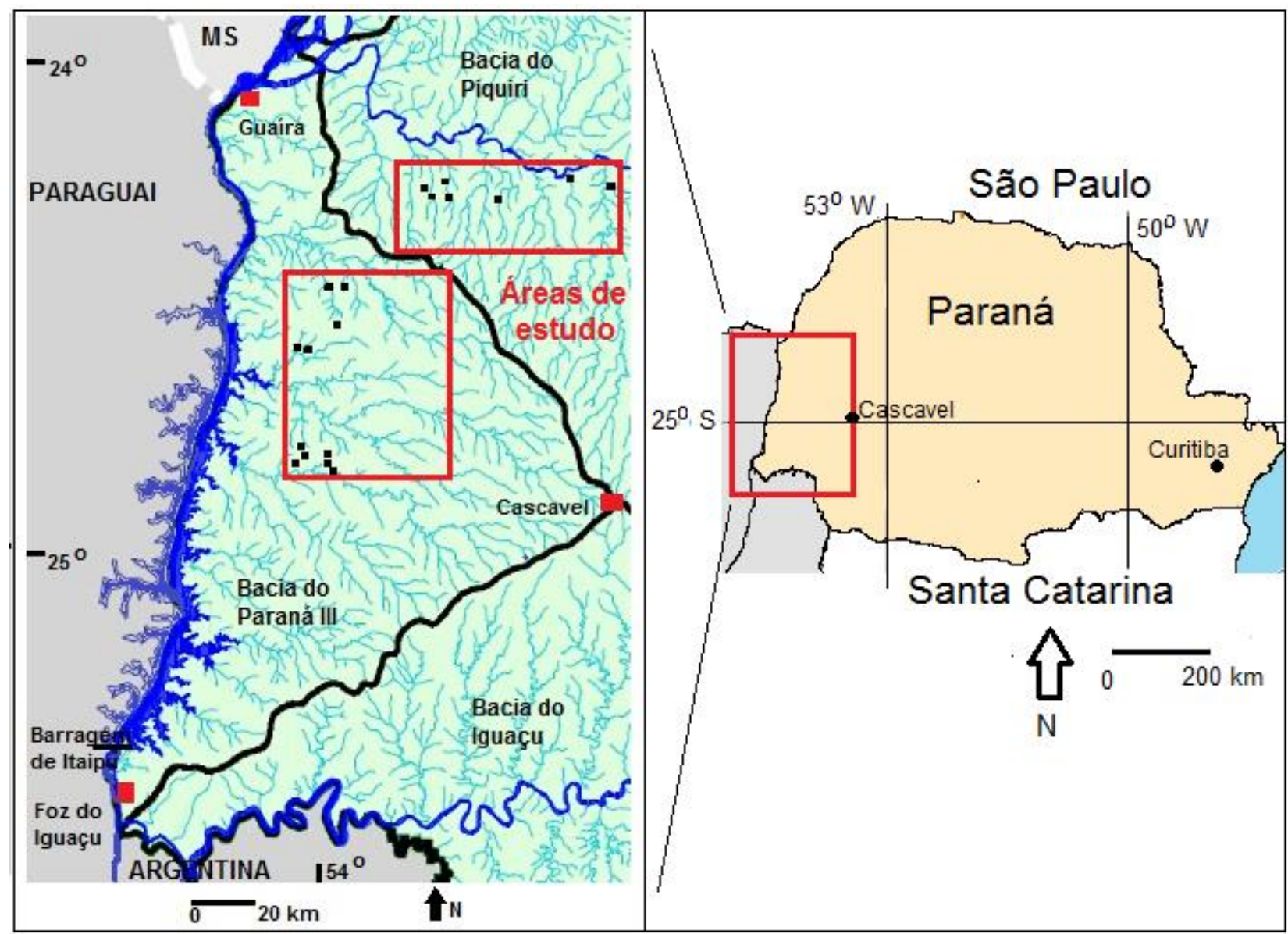

Figura 1: Delimitação das áreas de estudo nas bacias dos rios Piquiri e Paraná III, Oeste do estado do Paraná. Fonte: autor.

O clima da região é do tipo Cfa (classificação climática de Köppen), subtropical, úmido, mesotérmico, com precipitação média anual que varia de 1400 a $2000 \mathrm{~mm}$ e temperatura média anual que oscila entre 19 e $23 \mathrm{C}^{0}$ (CAVIGLIONE et al.; 2000) (Figura 2).

A colonização do extremo Oeste do Paraná começou na primeira metade do século XX com a Compañia de Maderas del Alto Paraná, empresa formada por ingleses residentes em Buenos Aires e visava a exploração de erva mate e madeira (FOWERAKER, 1982). As terras pertencentes a esta companhia, foram adquiridas pela imobiliária gaúcha Industrial Madeireira e Colonizadora Rio Paraná S.A. (MARIPÁ) em 1946 (WACHOWICZ, 1987). A venda das terras para colonizadores catarinenses e gaúchos começou em 1949, permitindo a ocupação sistemática da região a partir de 1951 (NIEDERAUER, 1955; SILVA et al., 1988 apud GÜTHS, 2000).

Os rios do oeste paranaense são típicos cursos fluviais de planalto, de caráter incisivo nos trechos superior e médio intercalando leitos aluviais e rochosos. Os trechos inferiores são caracterizados por canais com planície de inundação mais desenvolvida com leitos aluviais (EWALD, 2013). O desmatamento e a subsequente expansão da agricultura intensiva a partir da década de 1960 (OLIVEIRA, 2013) iniciou um gradual assoreamento dos canais fluviais (PFLUCK, 2009) alterando as características físicas e sedimentológicas originais dos cursos de água.

\section{O SISTEMA DE CLASSIFICAÇÃO DE ROSGEN}

A classificação de Rosgen (1994) é constituída por quatro níveis de detalhamento: I) Caracterização morfológica básica; II) Descrição morfológica; III) Condição geral do córrego e IV) Verificação. Neste artigo, dar-se-á ênfase aos dois primeiros níveis da classificação.

A classificação dos trechos fluviais é realizada utilizando os seguintes parâmetros: padrão fluvial, grau de entrincheiramento do canal, relação largura/profundidade, índice de sinuosidade, declividade da lâmina d'água e tipo de material de fundo. No nível I, a proposta de Rosgen discrimina nove tipos de canais, a partir 
da utilização dos cinco primeiros parâmetros acima citados (Quadro 1). No nível II, a inclusão do tipo de material de fundo e a divisão da declividade da lâmina d'água em duas ou três subcategorias, permitem a classificação dos canais em 94 subtipos (Figura 3).

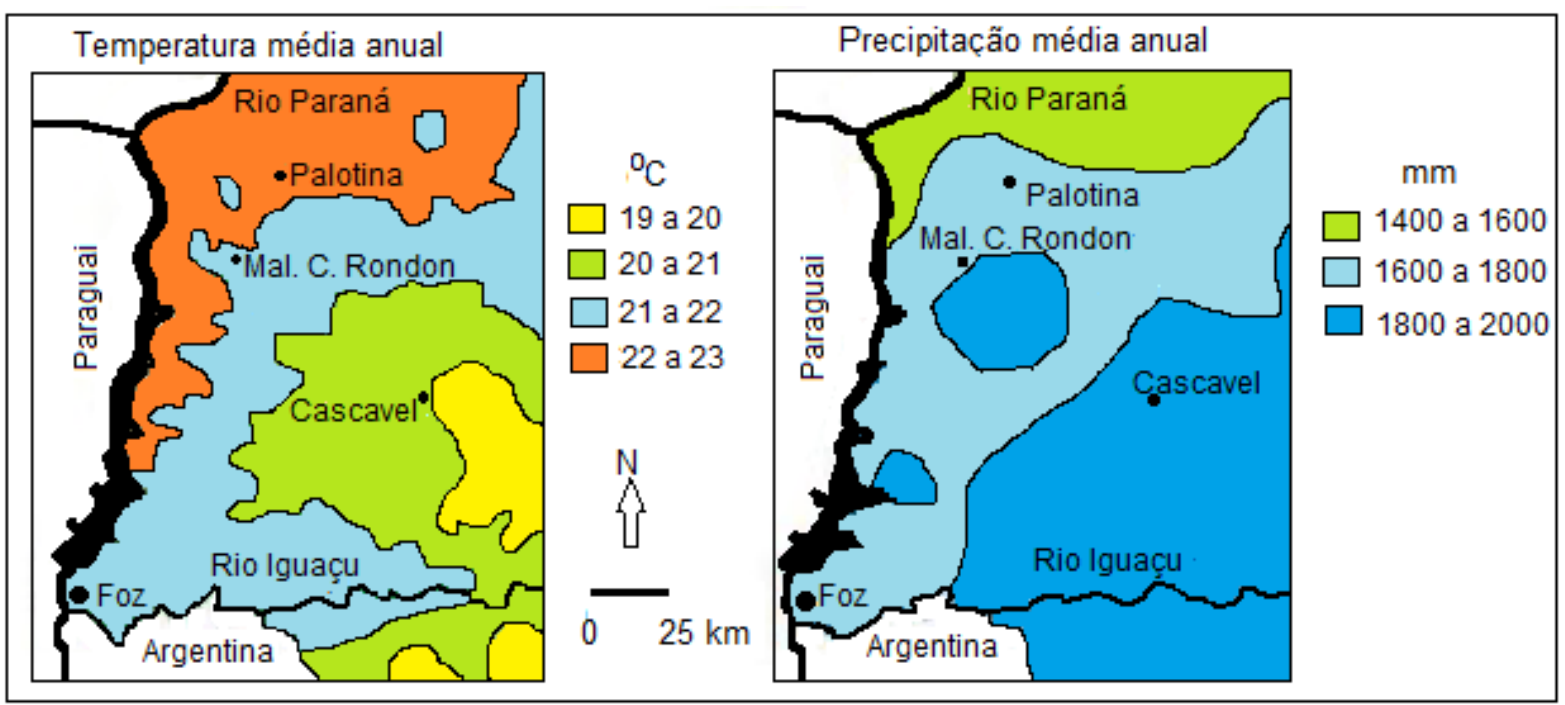

Figura 2: Variação da temperatura e precipitação na região Oeste do Paraná. Fonte: adaptada de Caviglione et al. (2000).

Quadro 1: Principais características dos tipos de canais classificados no nível I. E=grau de entrincheiramento do canal; $\mathrm{L} / \mathrm{P}=$ relação largura/profundidade; $\mathrm{S}=$ índice de sinuosidade e $\mathrm{D}=$ declividade da lâmina d'água. $\mathrm{NA}=$ não aplicado.

\begin{tabular}{|c|c|c|c|c|c|c|}
\hline Padrão fluvial & $\mathbf{E}$ & $\mathbf{L} / \mathbf{P}$ & $\mathbf{S}$ & $\mathrm{D}(\mathbf{m} / \mathbf{m})$ & $\begin{array}{c}\text { Tipo de } \\
\text { canal }\end{array}$ & Descrição \\
\hline \multirow[b]{2}{*}{ Multicanal } & $\mathrm{Na}$ & $>40$ & $\mathrm{Na}$ & $<0,04$ & $\mathrm{D}$ & $\begin{array}{l}\text { Canal entrelaçado com barras longitudinais e } \\
\text { transversais. Canal largo com margens } \\
\text { instáveis. }\end{array}$ \\
\hline & $>4,0$ & $<40$ & Variável & $<0,005$ & DA & $\begin{array}{l}\text { Canal anastomosado, com canais estreitos e } \\
\text { profundos, com vegetação ripária bem } \\
\text { desenvolvida. Relevo suave e canais com forte } \\
\text { sinuosidade. Margens estáveis. }\end{array}$ \\
\hline \multirow{7}{*}{ Unicanal } & $<1,4$ & $<12$ & $1,0-1,1$ & $>0,10$ & $\mathrm{Aa}+$ & $\begin{array}{l}\text { Canal com forte gradiente e entalhado, } \\
\text { disponibilidade de sedimentos grossos para } \\
\text { transporte. }\end{array}$ \\
\hline & $<1,4$ & $<12$ & $1,0-1,2$ & 0,04 a 0,10 & A & $\begin{array}{l}\text { Canal entalhado com forte declividade e } \\
\text { sequência de cascatas e degrau-depressão. } \\
\text { Ambiente de alta energia. Estável quando o } \\
\text { leito é formado por rocha ou capeado por } \\
\text { sedimentos rudáceos. }\end{array}$ \\
\hline & $<1,4$ & $<12$ & $>1,2$ & 0,02 a 0,039 & G & $\begin{array}{l}\text { Canal entalhado com voçorocamento, } \\
\text { sequência de degrau-depressão, baixa relação } \\
\text { largura/profundidade e gradiente moderado. }\end{array}$ \\
\hline & $<1,4$ & $>12$ & $>1,4$ & $<0,02$ & $\mathrm{~F}$ & $\begin{array}{l}\text { Canal meândrico com seqüência soleira- } \\
\text { depressão e com forte entalhamento, baixo } \\
\text { gradiente e alta relação largura/profundidade. }\end{array}$ \\
\hline & $1,4-2,2$ & $>12$ & $>1,2$ & 0,02 a 0,039 & B & $\begin{array}{l}\text { Canal moderadamente entalhado, declive } \\
\text { moderado, predomínio de soleiras. Margens } \\
\text { estáveis. }\end{array}$ \\
\hline & $>2,2$ & $<12$ & $>1,5$ & $<0,02$ & $\mathrm{E}$ & $\begin{array}{l}\text { Canal com baixo gradiente, padrão meândrico, } \\
\text { com sequência de soleira e depressão, baixa } \\
\text { relação largura/profundidade e baixa taxa de } \\
\text { deposição. }\end{array}$ \\
\hline & $>2,2-$ & $>12$ & $>1,2$ & $<0,02$ & $\mathrm{C}$ & $\begin{array}{l}\text { Baixo gradiente, canal meândrico, barras de } \\
\text { pontal, sequência de soleiras e depressões, } \\
\text { planície aluvial bem desenvolvida. }\end{array}$ \\
\hline
\end{tabular}

Fonte: Rosgen (1994) 


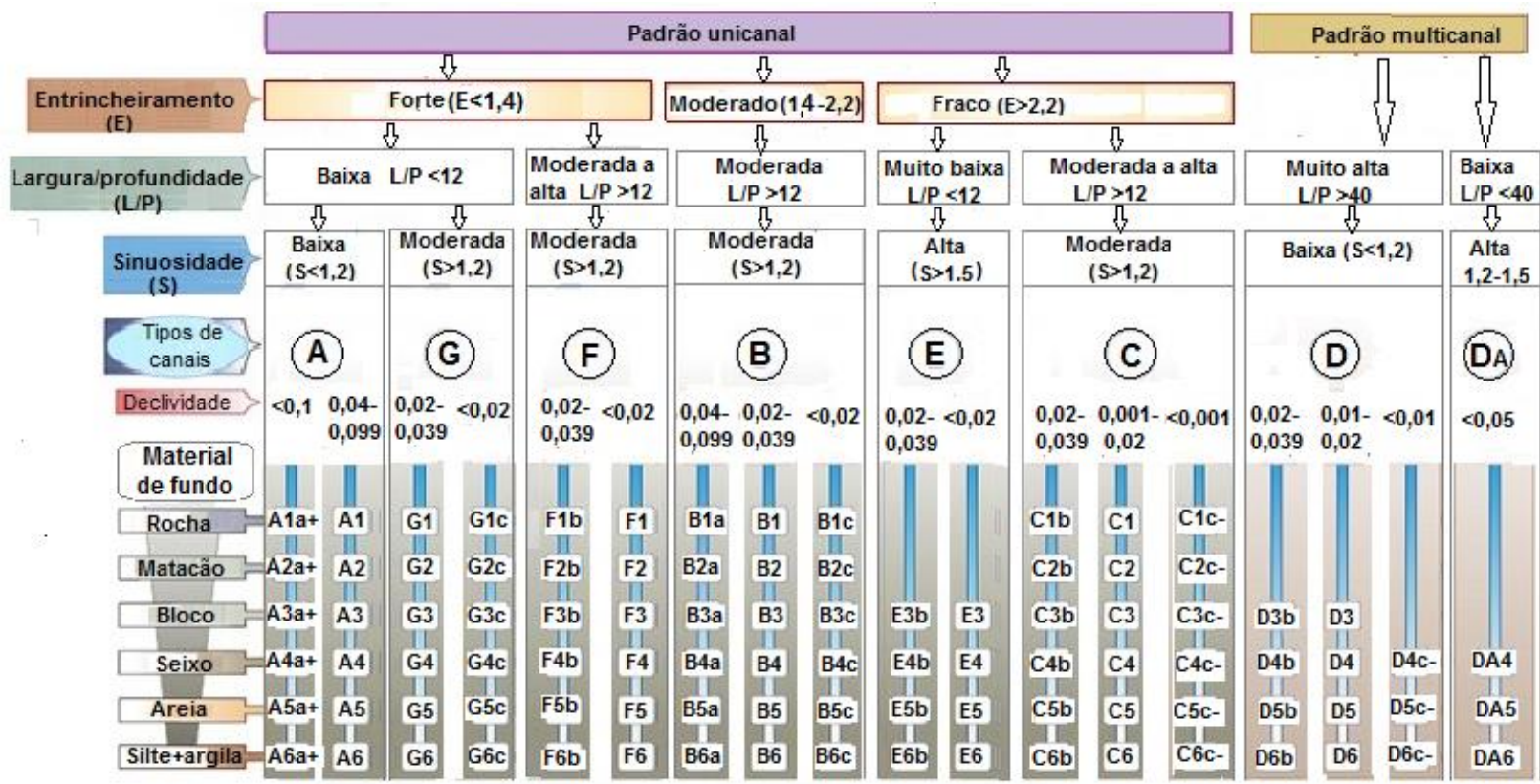

Figura 3: Tipos de canais definidos pela classificação de Rosgen no nível II. Fonte: Rosgen (1994).

Os parâmetros como grau de entrincheiramento do canal e relação largura/profundidade devem ser obtidos tendo como referência o nível de margens plenas. Por essa razão, a correta identificação deste nível é de fundamental importância na aplicação da classificação de Rosgen. A seguir, é realizada uma breve descrição dos parâmetros utilizados na classificação alfanumérica de Rosgen.

Padrão fluvial: é o primeiro elemento classificatório aplicado nas tabelas de Rosgen (Quadro 1, Figura 3). Assim, são definidos os tipos de canais com padrão unicanal (Aa+, A, B, C, E, F e G) e com padrão multicanal (Grupos D e DA).

Grau de entrincheiramento do canal (E): este parâmetro é definido pela relação entre a largura do canal em nível de margens plenas duplicado (flood-prone level) (Wm) e a largura do canal em nível de margens plenas (L) (Figura 4). Rosgen afirma que o nível Wm é atingido por cheias com até 50 anos de recorrência. $\mathrm{Na}$ seção de referência, deve ser levantado um perfil transversal detalhado, identificando nele o nível de margens plenas. O nível de Wm é obtido duplicando o valor da profundidade do canal em nível de margem plena (P) (Figura 3). Rosgen considera o entrincheiramento fraco quando $E>2,2$ e forte quando $E<1,4$. $O$ levantamento do perfil transversal deve ser estendido até o nível de terraço em ambas as margens.

Relação largura/profundidade (L/P): os valores da largura e da profundidade do canal em nível de margens plenas são mensurados em perfis transversais confeccionados a partir de levantamento topográfico convencional (Figura 4).

Índice de sinuosidade (S): este parâmetro é definido pelo quociente do comprimento do canal pelo comprimento do vale fluvial. Quando não se dispõe de uma carta topográfica detalhada da área de estudo (Escala>1/10.000), o traçado do canal deve ser obtido através de levantamento topográfico.

Declividade da lâmina d'água (D): A declividade é obtida dividindo o desnível da lâmina d'água no trecho considerado pelo comprimento do canal no mesmo trecho. A declividade deve ser medida através de levantamento topográfico ao longo de num trecho equivalente a 20 vezes à largura do canal em nível de margens plenas (LEOPOLD, 1994).

Tipo de material de fundo: os sedimentos são caracterizados a partir de análise granulométrica convencional de sedimentos arenosos (peneiramento) e lamosos (pipetagem). Quando predomina sedimentos rudáceos, a caracterização sedimentológica pode ser realizada com a medição direta do diâmetro das partículas selecionadas aleatoriamente no leito (WOLMAN, 1954) empregando paquímetro ou cascalhometro (FERNANDEZ; ARNDT, 2008). Na classificação de Rosgen é utilizada como parâmetro a classe textural na qual está inserida a mediana da distribuição $\left(\mathrm{D}_{50}\right)$ e recebe um número de identificação a ser utilizado na nomenclatura de classificação: 1=leito rochoso, 2=matacão, 3=bloco, 4=seixo, 5=areia 6=silte+argila (Figura 3). 


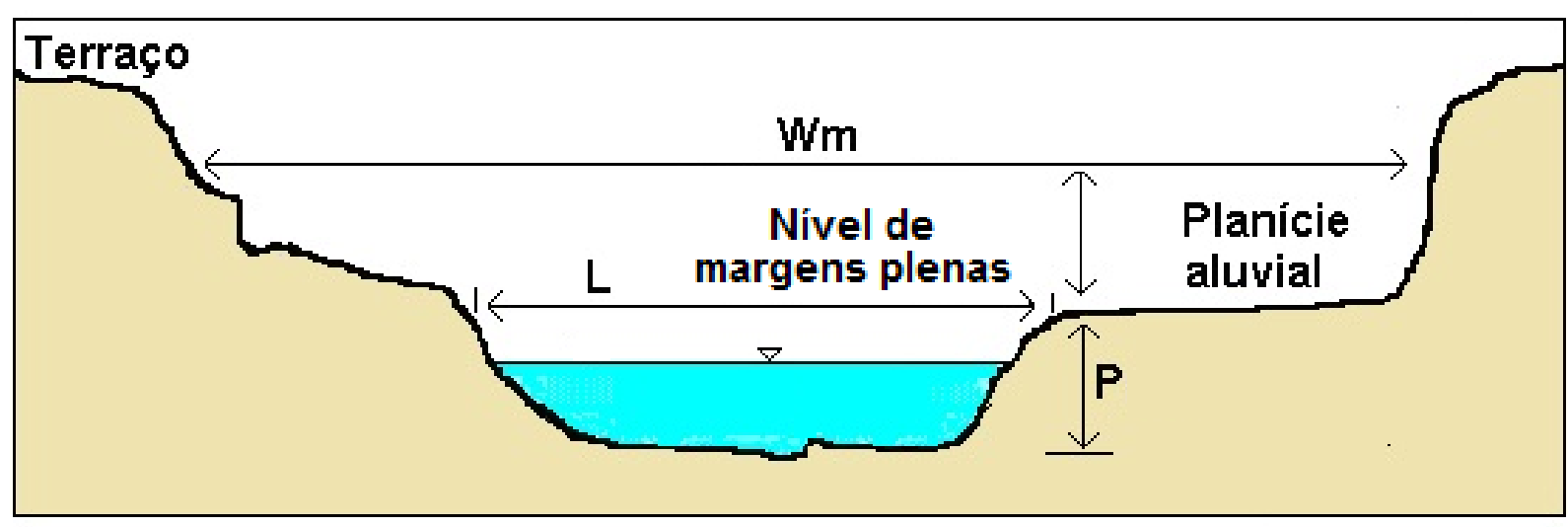

Figura 4: Elementos geométricos do canal empregados na classificação de Rosgen. As variáveis são medidas tendo como referência o nível de margens plenas. L=largura; $\mathrm{P}=$ profundidade e $\mathrm{Wm}=$ largura do canal em nível de margens plenas duplicado. Fonte: autor.

Os nove tipos de canais definidos no nível I são reclassificados no nível II em 94 subtipos mediante a inclusão do tipo de material de fundo e a divisão da declividade da lâmina d'água em duas ou três subcategorias (Figura 3). Na aplicação da classificação no nível II na Figura 3, podem ser necessários ajustes em razão ao "continuum" das variáveis morfológicas dos canais. No caso do grau de entrincheiramento (E) e do índice de sinuosidade (S) podem ser aceitos valores de $\pm 0,2$ para os limites tabelados destes parâmetros e de $\pm 2,0$ para a relação largura/profundidade $(\mathrm{L} / \mathrm{P})$. Por exemplo, um trecho com $\mathrm{E}=2,1$ e $\mathrm{L} / \mathrm{P}=10$ pode ser classificado no tipo E ampliando o valor de E para 2,2 e assim inserir o trecho em apreço na categoria E $>2,2$ para classificar no tipo E.

\section{CONCEITO E IDENTIFICAÇÃO DO NÍVEL DE MARGENS PLENAS}

A definição do nível de margens plenas e da descarga líquida correspondente é de grande importância prática e científica. Para fins práticos (construção de pontes, proteção de benfeitorias, etc.) a identificação da vazão de margens plenas é fundamental, pois indica o início da inundação (HENDERSON, 1961). Em termos científicos, a descarga de margens plenas representa a vazão que controla as dimensões do canal através dos processos erosivos e deposicionais. Fernandez (2003) comentou e exemplificou os principais critérios utilizados na identificação do nível de margens plenas.

A correta identificação deste nível é imprescindível na aplicação da classificação de Rosgen. O nível de margens plenas corresponde a um plano horizontal no qual a descarga líquida preenche na medida justa o canal fluvial antes de extravasar em direção a planície de inundação ativa (WOLMAN e LEOPOLD, 1957). Esta planície é definida como uma superfície plana adjacente ao canal fluvial, modelada pela ação erosiva ou deposicional do fluxo das cheias e inundada pelo menos uma vez a cada dois anos. O nível de margens plenas demarca o limite entre os processos fluviais que moldam o canal e os que constroem a planície de inundação. O terraço constitui uma superfície raramente inundada e representa a antiga planície de inundação.

Diversos métodos são utilizados para identificar o nível de margens plenas. Este nível pode ser identificado com base no reconhecimento de superfícies deposicionais, características botânicas e sedimentológicas e, aspectos morfológicos da seção transversal do canal. A superfície do fundo de vale foi adotada como referência por Nixon (1959), Woodyer (1968) entre outros. O nível médio das partes mais altas das barras de canal é outra referência utilizada (WOLMAN; LEOPOLD, 1957; HICKIN, 1968; LEWIS; McDONALD, 1973). O nível superior de deposição de sedimentos tamanho areia num canal foi adotado por Nunnally (1967) e, Leopold e Skibitzke (1967). O nível inferior de desenvolvimento da vegetação perene é um critério empregado por Schumm (1960), Nunnally (1967), Leopold (1994) e Radecki-Pawlik (2002). O nível de margens plenas também pode ser identificado a partir da análise da forma da seção transversal. Wolman (1955), Harvey (1969) e, Pickup e Warner (1976) adotaram a relação mínima da razão largura/profundidade num perfil transversal.

Quando a planície aluvial é bem desenvolvida, esta superfície foi adotada como referência na definição do nível de margens plenas (Figura 5A). Nos canais entalhados, o nível de margens plenas foi demarcado a partir da cota das barras de canal (Figura 5B). 

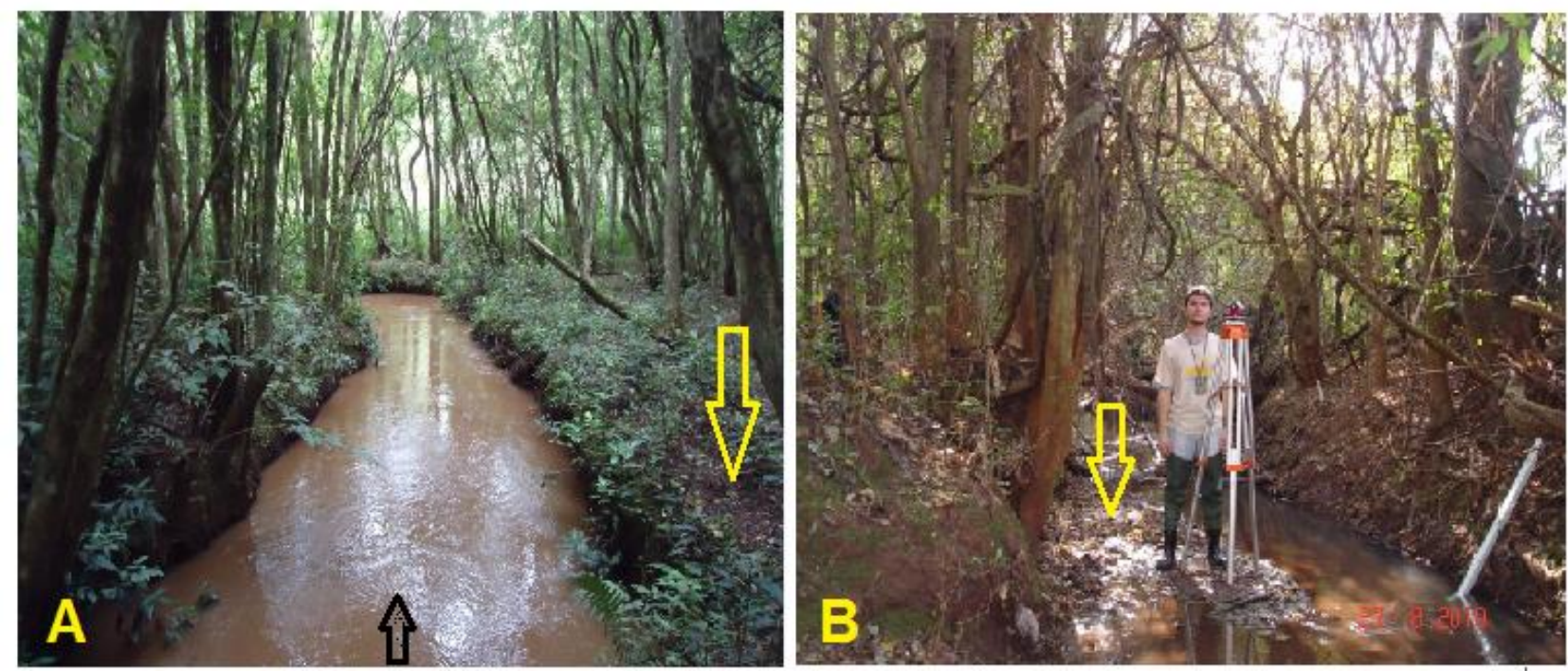

Figura 5: Definição da cota de margens plenas. A) canais com planície aluvial desenvolvida (córrego Aurora, ponto 3, fevereiro/2011). B) canais entrincheirados (córrego Jequitiba, ponto 5, agosto/2010). As setas indicam as superfícies adotadas para definir a cota de margens plenas. Fonte: autor.

\section{APLICAÇÃO DA CLASSIFICAÇÃO DE ROSGEN}

A classificação de Rosgen foi aplicada em 18 trechos fluviais distribuídos em seis municípios da região Oeste do Paraná (Figura 6). No Quadro 2 são elencados, além os dados de localização e área da bacia à montante do trecho estudado, os dados necessários para a aplicação da classificação no nível II [grau de entrincheiramento (E), relação largura/profundidade (L/P), índice de sinuosidade (S), declividade da lâmina d'água (D), tipo de material de fundo] e, finalmente a classificação dos trechos estudos.

A classificação foi aplicada de maneira satisfatória em 12 trechos selecionados (67\%) e agrupados nos tipos E e G, porém em seis pontos não foi possível aplicar a classificação (Quadro 2). A seguir, são enumeradas e comentadas as dificuldades encontradas na aplicação da proposta de Rosgen.

Em oito trechos, a classificação foi aplicada sem nenhum tipo de inconveniente (trechos 3, 6, 7, 8, 10, 13, 15 e 18). Estes pontos, classificados a maioria no tipo E, são trechos com planície aluvial desenvolvida, fraco grau de entrincheiramento, baixa relação $\mathrm{L} / \mathrm{P}$, alto índice de sinuosidade e declividade da lâmina d'água em torno ou inferior a $0,02 \mathrm{~m} / \mathrm{m}$. O único canal agrupado no tipo $\mathrm{Gc}$ (ponto 8) é caracterizado por possuir canal profundamente entalhado $(\mathrm{E}<1,4)$, relação $\mathrm{L} / \mathrm{P}<12$, índice de sinuosidade $>1,2$ e declividade da lâmina d'água $<0,02 \mathrm{~m} / \mathrm{m}$.

Em quatro trechos $(4,5,11$ e 17), os valores da sinuosidade foram inferiores aos tabelados, ou seja 1,5 para os tipos E e, 1,2 para o tipo G (Figura 3). Aplicando o conceito do "continuum" das variáveis morfológicas para a sinuosidade, os novos limites passam a ser de 1,3 para o tipo E e 1,0 para o tipo G. Desta forma, os trechos supracitados foram classificados nos tipos E e G (Quadro 2).

Nos seis trechos restantes não foi possível aplicar a classificação de Rosgen. Nestes pontos foram constatadas duas situações, identificadas com as siglas NA1 e NA2 (Quadro 2). Na situação NA1, os pontos $1,2,9,12$ apresentaram sinuosidade inferior ao valor de tabela, mesmo após rebaixar o limite da sinuosidade para 1,3 com a aplicação do conceito do "continuum" (Quadro 2). A mesma dificuldade foi encontrada por Savery et al. (2001) nos rios da Floresta Nacional Chequamegon-Nicolet (EUA), onde 10,7 \% dos trechos estudados apresentaram índices de sinuosidade abaixo do limite de tolerância.

Nos últimos dois trechos (14 e 16) a aplicação da classificação ainda foi mais difícil (situação NA2). No ponto14, os valores de E e L/P encaminham a classificação para o tipo E, porém a baixa sinuosidade $(1,04)$ e a presença de leito rochoso, não permitem classificar o ponto no tipo E. Por sua vez, no trecho 16 se repetem os mesmos inconvenientes verificados no ponto anterior e somando o fato da declividade $(0,047)$ se situar acima do valor máximo de tabela $(0,039)$ (Quadro 1).

Dentre os 18 trechos estudados, em nove pontos a sinuosidade foi inferior aos valores de tabela da classificação. Os trechos 1, 2 e 5 (bacia do Piquiri) e os pontos 9, 11, 12, 14, 16 e 17 na bacia do Paraná III 
(Figura 6) apresentaram esta característica. O alto número de trechos analisados com baixa sinuosidade pode ser consequência da influência de lineamentos tectônicos presentes no planalto basáltico da Bacia do Paraná (LIMA e BINDA 2013).

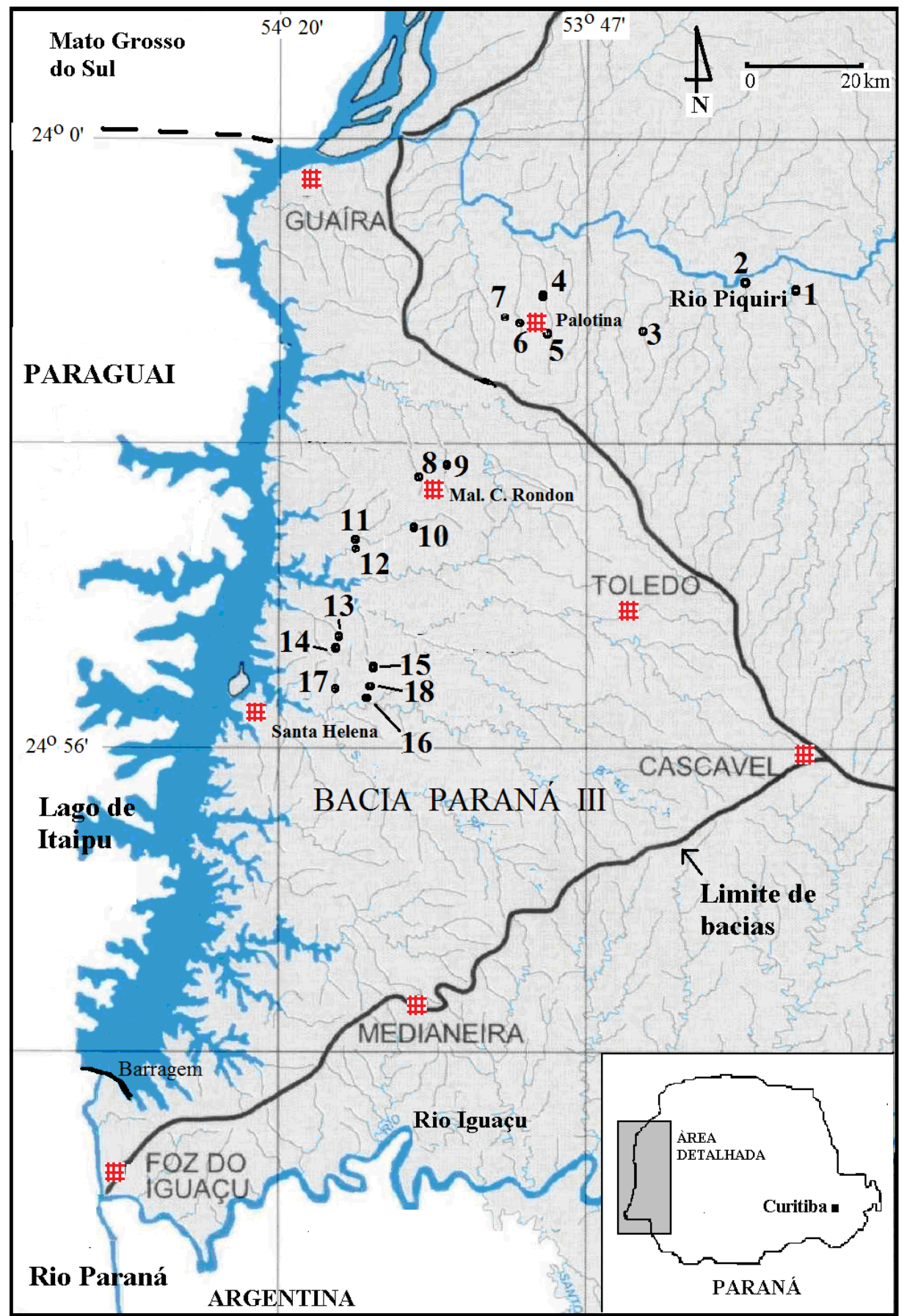

Figura 6: Localização dos trechos fluviais nas bacias dos Piquiri e Paraná III classificados pela proposta de Rosgen. Fonte: IAPAR (1994).

\section{LIMITAÇÕES DA CLASSIFICAÇÃO DE ROSGEN}

A classificação de Rosgen se baseia na correta identificação do nível de margens plenas. Esta dependência acarreta duas inconveniências. A primeira se refere à utilização da classificação por técnicos e pesquisadores pouco familiarizados com a dinâmica fluvial; isto gera identificações imprecisas do nível de margens plenas. A segunda, diz respeito a dificuldade de identificação do nível de margens plenas em bacias ou trechos afetados por intensas atividades antrópicas. A ocupação intensiva das bacias hidrográficas altera o regime hidrológico e a produção de sedimentos, acarretando mudanças na geometria do canal fluvial e nos processos erosivos e deposicionais na calha e na planície de inundação. Nestas condições, os critérios morfológicos adotados podem indicar o nível de margens plenas correspondente a tempos pretéritos 
Os críticos da proposta de Rosgen (MILLER e RITTER, 1996; GILLILAN, 1996; ASHMORE, 1999; JURACEK e FITZPATRICK, 2003; SIMON et al., 2005, LAVE, 2009 entre outros) concordam que a classificação só pode ser adotada objetivando a descrição dos cursos d'água e o fomento da troca de informações entre estudiosos. Estas metas são alcançadas nos níveis I e II. Os autores citados acima criticam o emprego dos níveis III e IV na elaboração de projetos de restauração ecológica dos rios para a qual é fundamental predizer o comportamento dos cursos fluviais. A classificação de Rosgen se baseia somente na descrição morfológica dos canais. Os críticos concordam que isto a torna inadequada na predição de respostas dos sistemas fluviais às perturbações naturais ou artificiais. Para tanto, devem ser incorporados na classificação parâmetros relacionados a dinâmica dos processos fluviais. Kondolf (2001), Kapitzke (2003) e Kochel (2005) citam exemplos da aplicação da classificação de Rosgen (níveis III e IV) em projetos de recuperação morfoecológica de canais fluviais com resultados indesejáveis.

Quadro 2: Aplicação do sistema de classificação de Rosgen (Nível II) no Oeste do Paraná. E=grau de entrincheiramento, $\mathrm{L}$ e $\mathrm{P}=$ largura e profundidade do canal medida em nível de margens plenas, $\mathrm{S}=$ índice de sinuosidade, $\mathrm{D}=$ declividade da lâmina d'água. Fonte: autor.

\begin{tabular}{|c|c|c|c|c|c|c|c|c|c|c|}
\hline $\mathbf{N}^{o}$ & $\begin{array}{c}\text { Córrego/Altitude/ } \\
\text { Munićpio/ } \\
\text { Coordenadas geográficas }\end{array}$ & $\begin{array}{c}\text { Área } \\
\left(\mathbf{k m}^{2}\right)\end{array}$ & $\mathbf{E}$ & $\mathbf{L}(\mathbf{m})$ & $\mathbf{P}(\mathbf{m})$ & $\mathbf{L} / \mathbf{P}$ & $\mathbf{S}$ & $\underset{(\mathbf{m} / \mathbf{m})}{\mathbf{D}}$ & $\begin{array}{l}\text { Material } \\
\text { de fundo }\end{array}$ & $\begin{array}{l}\text { Classif. } \\
\text { Rosgen }\end{array}$ \\
\hline 1 & $\begin{array}{l}\text { Araras/270 m/Formosa do Oeste } \\
24^{\circ} 16^{\prime} 15^{\prime} \text { 'S e } 53^{\circ} 27^{\prime} 06^{\prime} \mathrm{W}\end{array}$ & 49,8 & $>2,2$ & 9,45 & 1,29 & 7,32 & 1,10 & 0,0097 & Bloco & NA1 \\
\hline 2 & $\begin{array}{c}\text { Baiano/350 m/Assis } \\
\text { Chateaubriand } \\
24^{\circ} 15^{\prime} 07^{\prime} \mathrm{S} \text { e } 53^{\circ} 32 \text { ' } 38^{\prime \prime} \mathrm{W}\end{array}$ & 69,8 & $>2,2$ & 7,2 & 1,39 & 5,18 & 1,20 & 0,0047 & Seixos & NA1 \\
\hline 3 & $\begin{array}{c}\text { Aurora/307 m/Palotina } \\
24^{\circ} 21^{\prime} 16^{\prime} \text { "S e } 53^{\circ} 44^{\prime} 15^{\prime \prime} \mathrm{W}\end{array}$ & 30,5 & $>2,2$ & 2,33 & 1,11 & 2,11 & 1,68 & 0,013 & Areia & E5 \\
\hline 4 & $\begin{array}{l}\text { Água Branca/26 m/Palotina } \\
24^{\circ} 13^{\prime} 02^{\prime} \text { 'S e } 53^{\circ} 49^{\prime} \quad 25^{\prime} \mathrm{W}\end{array}$ & 5,2 & $>2,2$ & 3,66 & 0,75 & 4,88 & 1,30 & 0,0099 & Areia & E5 $(*)$ \\
\hline 5 & $\begin{array}{c}\text { Jequitiba/285 m/Palotina } \\
24^{\circ} 10^{\prime} 55^{\prime} \mathrm{S} \text { e } 53^{\circ} 49^{\prime} 20^{\prime} \mathrm{W}\end{array}$ & 2,5 & 1,3 & 3,06 & 0,75 & 4,08 & 1,10 & 0,016 & Areia & G5c $(*)$ \\
\hline 6 & $\begin{array}{c}\text { Santa Fé } / 283 \mathrm{~m} / \text { Palotina } \\
24^{\circ} 18^{\prime} 0 \text { " “ } \mathrm{Se} \text { e } 53^{\circ} 51^{\prime} 04 \text { ” } \mathrm{W}\end{array}$ & 28,9 & $>2,2$ & 4,43 & 1,3 & 3,41 & 1,80 & 0,0017 & Areia & E5 \\
\hline 7 & $\begin{array}{c}\text { Quati/292 m/Palotina } \\
24^{\circ} 18^{\prime} 28^{\prime} \text { 'S e } 53^{\circ} 54^{\prime} 22^{\prime \prime} \mathrm{W}\end{array}$ & 6,6 & $>2,2$ & 2,08 & 0,57 & 4,95 & 1,50 & 0,011 & Areia & E5 \\
\hline 8 & $\begin{array}{l}\text { Guavirá/330 m/Mal. C. Rondon } \\
24^{\circ} 31^{\prime} 17^{\prime \prime} \text { S e } 54^{\circ} 04^{\prime} 24^{\prime \prime} \mathrm{W}\end{array}$ & 10,7 & 1,1 & 5,57 & 0,63 & 8,84 & 1,30 & 0,0052 & Areia & G5c \\
\hline 9 & $\begin{array}{l}\text { Guará/339 m/Mal. C. Rondon } \\
24^{\circ} 30^{\prime} 58^{\prime \prime} \text { S e } 54^{\circ} 01^{\prime} 17^{\prime \prime} \mathrm{W}\end{array}$ & 13,6 & $>2,2$ & 5,24 & 1,90 & 2,76 & 1,21 & 0,0015 & $\begin{array}{l}\text { Silte+ } \\
\text { Argila }\end{array}$ & NA1 \\
\hline 10 & $\begin{array}{c}\text { Concórdia/270 m/Mal. C. } \\
\text { Rondon } \\
24^{\circ} 36^{\prime} 54^{\prime} \text { S S e } 54^{\circ} 06^{\prime} 26 \mathrm{~W}\end{array}$ & 2,2 & $>2,2$ & 2,26 & 0,59 & 3,83 & 1,60 & 0,0054 & Areia & E5 \\
\hline 11 & $\begin{array}{l}\text { Arroio Fundo/235 m/Mal. C. } \\
\text { Rondon } \\
24^{\circ} 38^{\prime} 06^{\prime} \text { 'S e } 54^{\circ} 11^{\prime} 32^{\prime} \text { W }\end{array}$ & 187,2 & $>2,2$ & 15,3 & 1,4 & 10,9 & 1,30 & 0,0018 & Seixo & E4 $(*)$ \\
\hline 12 & $\begin{array}{c}\text { São João / } 235 \text { m/ Mal. C. } \\
\text { Rondon } \\
24^{\circ} 38^{\prime} 35^{\prime \prime} \text { S e } 54^{\circ} 11^{\prime} 18^{\prime \prime} \mathrm{W}\end{array}$ & 5,5 & $>2,2$ & 3,17 & 1,04 & 3,05 & 1,20 & 0,0005 & $\begin{array}{l}\text { Silte+ } \\
\text { Argila }\end{array}$ & NA1 \\
\hline 13 & $\begin{array}{l}\text { Golondrina / } 282 \mathrm{~m} / \mathrm{Santa} \\
\text { Helena } \\
24^{\circ} 45^{\prime} 19^{\prime} \text { S S e } 54^{\circ} 12^{\prime} 10^{\prime \prime} \mathrm{W}\end{array}$ & 2,2 & $>2,2$ & 2,0 & 0,76 & 2,63 & 1,55 & 0,0062 & Areia & E5 \\
\hline 14 & $\begin{array}{l}\text { Volta Seca } / 304 \mathrm{~m} / \text { Santa Helena } \\
24^{\circ} 46^{\prime} 37^{\prime} \text { 'S e } 54^{\circ} 12 \text { ' } 31^{\prime} \text { W }\end{array}$ & 0,88 & $>2,2$ & 3,54 & 0,40 & 8,85 & 1,04 & 0,026 & Rocha & NA2 \\
\hline 15 & $\begin{array}{l}\text { Barra Funda/319 m/ São José } \\
\text { das Palmeiras } \\
24^{\circ} 48^{\prime} 20^{\prime \prime} \text { S e } 54^{\circ} 08^{\prime} 57^{\prime \prime} \mathrm{W}\end{array}$ & 3,6 & $>2,2$ & 4,17 & 0,60 & 6,95 & 2,15 & 0,017 & Seixo & E4 \\
\hline 16 & $\begin{array}{l}\text { Abelha/275 m/São José das } \\
\text { Palmeiras } \\
24^{\circ} 49^{\prime} 26^{\prime \prime S} \text { e } 54^{\circ} 06^{\prime} 58^{\prime \prime} \mathrm{W}\end{array}$ & 1,43 & $>2,2$ & 4,2 & 0,46 & 9,13 & 1,08 & 0,047 & Rocha & NA2 \\
\hline 17 & $\begin{array}{l}\text { Ponte Quebrada/233 m/Santa } \\
\text { Helena } \\
24^{\circ} 49^{\prime} 42^{\prime \prime} \text { e } 54^{\circ} 16^{\prime} 16^{\prime} \mathrm{W}\end{array}$ & 24,2 & $>2,2$ & 9,3 & 1,36 & 6,84 & 1,3 & 0,0099 & Seixo & E4 $\left(^{*}\right)$ \\
\hline 18 & $\begin{array}{c}\text { Sanga Nova/267 m/São Jose das } \\
\text { Palmeiras } \\
24^{\circ} 50^{\prime} 47^{\prime} \text { 'S e } 54^{\circ} 13^{\prime} 29{ }^{\prime \prime} \mathrm{W}\end{array}$ & 1,63 & $>2,2$ & 2,0 & 0,47 & 4,25 & 1,76 & 0,021 & Seixo & $\mathrm{E} 4 \mathrm{~b}$ \\
\hline
\end{tabular}

Notas: (*) a classificação foi adotada aplicando os critérios do "continuum" das variáveis morfológicas. NA1 e NA2 classificação não aplicada. 
Os críticos da classificação chamam a atenção sobre duas fontes de erros: a aplicação indevida da classificação e sua utilização por técnicos não capacitados. A primeira se refere ao uso da proposta apenas como instrumento de descrição morfológica e sedimentológica dos rios e a segunda aborda a aplicação da classificação por técnicos que desconheçam os processos de dinâmica fluvial. Neste último caso, os possíveis erros podem ser originados não somente nas medições de elementos lineares do canal como largura, declividade, etc., mas também na identificação incorreta do nível de margens plenas.

\section{CONSIDERAÇÕES FINAIS}

O trabalho mostrou a aplicação da classificação fluvial de Rosgen em trechos fluviais localizados no planalto basáltico, extremo Oeste do Paraná. Doze dos dezoito trechos estudados (67\%) foram classificados nos tipos E e G. Os canais pertencentes ao tipo E são caracterizados pelo baixo gradiente, padrão meândrico, com seqüência de soleiras e depressões e baixa relação largura/profundidade, enquanto que os canais do tipo G são entalhados, apresentam gradientes baixos a moderados e baixa relação largura/profundidade. Nos seis canais restantes não foi possível aplicar a classificação de Rosgen, pela falta de enquadramento das variáveis morfológicas dentro dos limites da classificação. Dentre as variáveis utilizadas pela classificação, a sinuosidade mostrou-se o parâmetro com menor compatibilidade na classificação de Rosgen. A sinuosidade não alcançou o limite mínimo estabelecido pelo sistema de classificação em $50 \%$ dos trechos analisados. Os baixos índices da sinuosidade dos canais podem ser provocados pela grande quantidade de lineamentos tectônicos presente no planalto basáltico da bacia do Paraná.

A classificação de Rosgen adota o nível de margens plenas como principal referência. A correta classificação dos canais depende da precisa identificação deste nível. Em rios entalhados ou alterados pela atividade humana, a difícil identificação da cota de margens plenas pode levar a escolha equivocada do nível afetando o resultado da classificação. Em suma, a classificação de Rosgen constitui um excelente instrumento para inventariar de maneira padronizada as condições morfológicas e sedimentológicas dos cursos d'água de uma determinada região.

\section{REFERENCIAS}

ALMEIDA NETO, P. Hidrogramas experimentais de áreas alagadas da microbacia do rio Jacupiranguinha, baixo Ribeira do Iguape, SP. Programa de Pós-Graduação em Hidráulica e Saneamento, Escola de Engenharia de São Carlos (USP), 2007. 174 p.

ANTÚNEZ,N.C. Criterios técnicos de delimitación y codificación de cuencas, caso específico región oriental del Paraguay. Secretaria del Ambiente (SEAM), Asunción - Paraguay, 26 p., 2011.

ASHMORE, P. What would we do without Rosgen?: rational regime equations and channel design. Proceeding of the Second International Conference on Natural Channel Systems, March, 1999, Niagara Falls, Canada, 1999. p. 343-349.

BRICE, J. Planform properties of meandering rivers. In: Elliott, C.M. (ed.) River meandering, Proceedings of Conference Rivers 83, New York: American Society of Civil Engineers, 1984. p. 1-15.

CASTRO, J.M. Stream classification in the Pacific Northwest: methodologies, regional analyses, and application. PhD Thesis, Oregon State University. 1997. 115 p.

CAVIGLIONE, J.H.; KIIHL, L.R.B.; CARAMORI, P.H.; OLIVEIRA, D.Cartas climáticas do Paraná. Londrina : IAPAR, 2000. CD

CHURCH, C. Channel morphology and typology. In: Callow, P. e Petts, G.E. (Eds.) The rivers handbook: hydrological and ecological principles, vol.1, Blackwell, Oxford. 1992. 'p. 126-143.

CRESPO, A., DAMME, P.; ZAPATA, M. Clasificación de cuencas de Bolivia según la metodología de Pfafstetter. Revista Boliviana de Ecologia y Conservación Ambiental, 22: 69-76, 2008.

DAVIS, W.M. The geographical cycle. Geography Journal, 14: 481-504. 1899.

ESPTEIN. C.M. Application of Rosgen analysis to the New Jersey Pine Barrens. Journal of the American Water Resources Association, 38 (1): 69-78. 2002.

ESTILIANO, E.O. Influencia da geomorfologia fluvial na distribuição espacial das assembléias de peixes do rio Paraíba do Sul. Dissertação de mestrado, Universidade Rural Federal do Rio de Janeiro, Instituto de Florestas, 2006. 56 p. 
EWALD, K.H. Caracterização de formas topográficas em fundos de vale no planalto de Cascavel, região Oeste do estado do Paraná. Dissertação de mestrado, Programa de Pós-Graduação em Geografia, Universidade Estadual do Oeste do Paraná, Marechal Cândido Rondon, 56 p. 2013.

FERNANDEZ, O.V.Q. Determinação do nível e da descarga de margem plena em cursos fluviais. Boletim de Geografia, Universidade Estadual de Maringá, 21 (1): 97-109. 2003.

FERNANDEZ, O.V.Q.; ARNDT, M.A. Comparação da eficácia do paquímetro e cascalhometro na análise granulométrica de sedimentos rudáceos. Anais...VIII Encontro Nacional de Engenharia de Sedimentos, Campo Grande (MS),12 p, CD-Rom. 2008.

FOWERAKER, J. A luta pela terra: a economia política da fronteira pioneira no Brasil em 1930 aos dias atuais. Rio de Janeiro, Ed. Zahar. 1982.

FRANCO-MAGALHÃES, A.O.B.; HACKSPACHER, P.C.; SAAD, A.R. Exumação tectônica e reativação de paleolineamentos no Arco de Ponta Grossa: termocronologia por traços de fissão em apatitas. Revista Brasileira de Geociências, 40 (2): 184-195. 2010.

GILLILAN, S. Use and misuse of channel classification schemes. Stream Notes, Technical Publication, Rocky Mountain Research. October 1996.

GOODWIN, C.N. Fluvial classification: neanderthal necessity or needless normalcy. Proceedings of Specialty Conference on Wildland Hydrology. Olson, D.S and Potyondy, J.P (Eds.) American Water Resources Association, Bozeman, MT, 1999. p. 229-236.

GÜTHS, L.D. Zona Bonita nos caminhos da colonização da fronteira. In: Marcos A. Lopes (Ed.) Espaços da Memória. Fronteiras. Edunioste, Cascavel (PR). 2000, p. 135-160.

HARVEY, A.M. Channel capacity and the adjustment of streams to hydrologic regime. Journal of Hydrology, 8: 82-98. 1969.

HENDERSON, F.M. Stability of alluvial channels. Journal of the Hydraulic Division, American Society of Civil Engineers 87: 109-138. 1961.

HEY, R.D. Fluvial geomorphology methodology for natural stable channel design. American Water Resources Association, 42: 357-374. 2006.

HICKIN, E.J. Channel morphology bankfull stage and bankfull discharge of streams near Sydney. Australia. Journal of Science, 30 (7): 274-275. 1968.

HORTON, R.E. Erosional development of stream and their drainage basins: hydro-physical approach to quantitative morphology. Bulletin of the Geological Society of America 56: 275-370. 1945.

IAPAR - Fundação Instituto Agronômico do Paraná. Cartas climáticas básicas do Estado do Paraná, Curitiba (PR), 1994. 49 p.

JONES, S.; CANDLER, J. Implementation of natural channel design on two Georgia Power stream restoration projects. Proceeding of the Georgia Water Resources Conference, April 25-27, 2005, University of Georgia, Athens, Georgia. 2005.

JURACEK, K.; FITZPATRICK, F. Limitations and implications of stream classification. American Water Resources Association, 39 (3): 659-670. 2003.

KAPITZKE, R. Appendix C(4): Natural Channel Design at the University of Guelph. In: Agencies, people and sites visited in Canada (Alberta and Ontario), Land and Water Australia Project JCU 15, Traveling Fellowship Report, 2003. p. 6-7.

KELLERHALS, R.; CHURCH, M.; BRAY, D.I. Classification and analysis of river processes. Journal of the Hydraulic Division, 102: 813-829. 1976.

KOCHEL, R.C.; MILLER, J.R.; LORD, M.; MARTIN, T. Geomorphic problems with in-stream structures using natural channel design strategy for stream restoration projects in North Carolina: Geological Society of America Abstracts with Programs, 37 (7), p. 329. 2005.

KONDOLF, G.M.; SMELTZER, M.W.; RAILSBACK, S.F. Design and performance of a channel reconstruction project in a coastal California gravel-bed stream. Environmental Management, 28 (6): 761 776. 2001. 
KONDOLF, G.M.; MONTGOMERY, D.R.; PIÉGAY, H.; SCHMITT, L. Geomorphic of rivers and streams. In: Kondolf, G.M. e Piégay, H. (Eds.). Tools in Fluvial Geomorphology. John Wiley \& Sons, Inc., 2003, p. 171-204.

LAVE, R. The controversy over natural channel design: substantive explanations and potential avenues for resolution. Journal of the American Water Resources Association, 45 (6): 1519-1532. 2009.

LEOPOLD, L.B. Hydrology for urban planning - A guide book on the hydrologic effects on urban land use. USGS Circ. 554, 1968, 18 p.

LEOPOLD, L.B. A view of the river. Harvard University Press. Cambridge, Massachusetts. 1994.

LEOPOLD, L.B.; WOLMAN, M.G. River channel patterns - braided, meandering and straigth. Profissional Paper, United Stated Geological Survey, 282B. 1957.

LEOPOLD, L.B. \& SKIBITZKE, H.E. Observation on unmeasured rivers. Geographical Annaler 49: 247-255. 1967.

LEWIS, C.P.; McDONALD, B.C. Rivers of the Yukon north slope. In: Fluvial Processes and Sedimentation, 1973, p. 251-271.

LIMA, A.G.; BINDA, A.L. Lithologic and structural control on fluvial knickzones in basalts of the Parana Basin, Brazil. Journal of South Americs Earth Sciences, 48: 262-270. 2013.

McCANDLESS, T.L. Maryland stream survey: bankfull discharge and channel characteristic of streams in the Allegheny Plateau and the valley and ridge hydrology regions. US Fish and Wildlife Service. Annapolis, Maryland, 2003, 33 p.

MIALL, A.D. The geology of fluvial deposits - sedimentary facies, basin analysis and petroleum geology. Springer-Verlag, Berlin, 1996, 582 p.

MILLER, J.R.; RITTER, J.B. An examination of the Rosgen classification of natural rivers. Catena 27: 295299. 1996.

MONTGOMERY, D.R; BUFFINGTON, J.M. Channel-reach morphology in mountain drainage basins. Geological Society of America Bulletin, 109: 596-611. 1997.

NARDY, A.J.R.; OLIVEIRA, M.A.F.; BETANCOURT, R.H.S.; VERDUGO, D.R.H.; MACHADO, F.B. Geologia e estratigrafia da Formação Serra Geral. Geociências (Unesp, São Paulo): 21: 15-32. 2002.

NIEDERAUER, O. Plano de colonização. Escritório da Maripá, junho de 1955. Toledo (PR). 1955.

NIXON, M. A study of bankfull discharge of rivers in England and Wales. In: Proceedings of the Institution of Civil Engineers, 12: 157-175. 1959.

NUNNALLY, N.R. Definition and identification of channel and overbank deposits and their respective roles in floodplain formation. Professional Geographer 19: 1-4. 1967.

OLIVEIRA, V.S.K. Implicações socioambientais causadas pelo processo de ocupação da região oeste do estado do Paraná entre as décadas de 1940 e 1980. Dissertação de mestrado, Programa de Pós-Graduação em Geografia, Universidade Estadual do Oeste do Paraná, Marechal Cândido Rondon, 125 p. 2013.

PFAFSTETTER, O. Classificação de bacias hidrográficas - Metodologia de codificação. Rio de Janeiro, RJ: Departamento Nacional de Obras de Saneamento (DNOS), p. 19. 1989.

PFLUCK, L.D. Riscos ambientais: enxurradas e desabamentos na cidade de Marechal Cândido Rondon-PR, 1980 a 2007. Tese (Doutorado em Geografia) - Universidade Federal de Santa Catarina, 274 p., 2009.

PICKUP, G.; WARNER, R.F. Effects of hydrologic regime on magnitude and frequency of dominant discharge. Journal of Hydrology, 29: 51-75. 1976.

POPOV, I.V. Hydromorphological principles of the theory of channel processes and their use in hydrotechnical planning. Soviet Hydrology, 2: 188-195. 1964.

RADECKI-PAWLIK, A. Bankfull discharge in mountain streams: theory and practice. Earth Surface Processes and Landforms, 27: 115-123. 2002. 
ROPER, B.B.; BUFFINGTON, J.M.; ARCHER, E.; MOYER, C.; WARD, M. The role of observer variation in determining Rosgen stream types in Northeastern Oregon mountain streams. Journal of the American Water Resources Association, 44 (2): 417-427. 2008.

ROSGEN, D.L. A stream classification system. In: Riparian Ecosystems and their management. First North American Riparian Conference. Rocky Mountains Forest and Range Experiment Station, RM-120, 1985, pp. 91-95.

ROSGEN, D.L. A classification of natural rivers. Catena, 22: 169-199. 1994.

ROSGEN, D.L. A stream channel stability assessment methodology. Proceedings of the Seventh Federal Interagency Sedimentation Conference, vol. 2, p 18-26, March 25-29, Reno, Nevada. 2001.

RUIZ, R.; TORRES, H.; AGUIRRE, M. Memoria descriptiva de la delimitación y codificación de unidades hidrográficas del Perú. INRENA. Lima. 2006.

RUST, B.R. A classification of alluvial channel systems. In: Miall, A.D. (Ed.) Fluvial Sedimentology. Canadian Society of Petroleum Geologists, Calgary, Alberta, Canada, 1978, p. 187-198.

SANTOS, L.J.C.; OKA-FIORI, C.; CANALI, N.E.; FIORI, A.P.; SILVEIRA, C.T.; SILVA, J.M.F.; ROSS, J.L.S. Mapeamento geomorfológico do estado do Paraná. Revista Brasileira de Geomorfologia, 7 (2): 3-12. 2006.

SAVERY, T.S.; BELT, G.H.; HIGGINS, D.A. Evaluation of the Rosgen stream classification system in Chequamegon-Nicolet National Park, Wisconsin. Journal of the American Water Resources Association, 37 (3): 641-654. 2001.

SCHUMM, S.A. The shape of alluvial channels in relation to sediment type. Professional Paper, United States Geological Survey, Washington, 1960, 352 p.

SCHUMM, S.A. A tentative classification of alluvial river channels. US Geological Survey Circular, 1963, $477 \mathrm{p}$.

SCHUMM, S.A. Evolution and response of the fluvial system, sedimentologic implications. SEPM Special Publications, 31: 19-29. 1981.

SIMON, A. A model of channel response in disturbed alluvial channels. Earth Surface Processes and Landforms, 14: 11-26. 1989.

SIMON, A.; DOYLE, M.; KONDOLF, M; SHIELDS Jr., F.; ROADS, GRANT, G.; FITZPATRICK, F.; JURACEK, K.; McPHILIPS, M. ; MACBROOM, J. How well do the Rosgen classification and associated "natural channel design" methods integrate and quantify fluvial processes and channel response?. Proceedings of the 2005 World Water and Environmental Resources Congress, May 15-19, 2005, Anchorage, Alaska.

STRAHLER, A.N. Quantitative geomorphology of drainage basins and channel networks. In: CHOW, Ven Te (Ed.) Handbook of applied hydrology: a compendium of water resources technology. New York: McGraw Hill, 1964. p. 4-39 - 4-75.

WACHOWICZ, R. Obrageros, mensus e colonos: história do oeste do Paraná. Curitiba, ed. Vicentina. 1987.

WARD, A.; D'AMBrosio, J.; MECKLENBURG, D. Stream Classification. Ohio State University. Engineering Fact Sheet, AEX 445-01, 2008, 8 p.

WHITING, P.J.; BRADLEY, J.B. A process-based classification system of headwater steams. Earth Surface Processes and Landforms, 18: 603-612. 1993.

WOLMAN, M.G. A method of sampling coarse bed material. Transactions of the American Geophysical Union, 35: 951-956. 1954.

WOLMAN, M.G. The natural channel of Brandywine creek. Pennsylvania. Professional Paper, United States Geological Survey, Washington, 271, 1955, 56 p.

WOLMAN, M.G.; LEOPOLD, L.B. River flood plains: some observations on their formation. Professional Paper, United States Geological Survey, Washington, 282-C. 1957.

WOODYER, K.D. Bankfull frequency in rivers. Journal of Hydrology, 6: 114-142. 1968.

WOOLFE, K.J; BALZARY, J.R. Fields in the spectrum of channel style. Sedimentology, 43: 797-805. 1996. 